\title{
Normas preliminares del Test de Desarrollo Psicomotor (TEPSI) para niños argentinos de 3 y 4 años
}

\section{Preliminary standards for the Psychomotor Development Test (TEPSI) for Argentine children aged 3-4}

\author{
Adriana V. L. Espósitoa, ${ }^{\mathrm{a},}$, Celina Graciela Korzeniowskia, ${ }^{\mathrm{ab}}$, Marina Santini Bertoldi ${ }^{\mathrm{a}}$

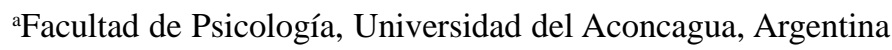 \\ ${ }^{b}$ Consejo Nacional de Investigaciones Científicas y Técnicas (CONICET), Argentina
}

\begin{abstract}
Resumen
El presente estudio tiene como objetivos elaborar normas locales del Test de Desarrollo Psicomotor (TEPSI) para niños argentinos y contrastar el nivel de desarrollo psicomotor y su velocidad de crecimiento entre la muestra argentina y la chilena de estandarización del instrumento. Se evaluaron 205 niños, entre 3 y 5 años de edad, que concurrían a jardines maternales públicos de la provincia de Mendoza. Se estimó la consistencia interna del test, se calcularon las correlaciones ítem-subtest, se empleó un ANOVA a priori, se calcularon las puntaciones T por grupo etario y se estimó la $d$ de Cohen para valorar la magnitud de las diferencias entre las muestras contrastadas. El TEPSI evidenció adecuadas propiedades psicométricas y presentó alta sensibilidad para valorar las diferencias de edad en el desarrollo psicomotor de los infantes. Se registró un desempeño psicomotor superior de los preescolares argentinos en comparación con sus pares chilenos y se observaron diferentes ritmos evolutivos entre ambos grupos. Estos resultados señalan la importancia de construir normas locales actualizadas del TEPSI que contemplen las características socioculturales de cada país, en miras a obtener una evaluación precisa y confiable del desarrollo psicomotor infantil.
\end{abstract}

Palabras clave: baremos, neurodesarrollo, niños, psicomotor, TEPSI.

\begin{abstract}
The goals of this study are to develop local standards for the Psychomotor Development Test (TEPSI) for Argentine children, and to contrast the level of psychomotor development and its growth rate between the Argentine and Chilean samples used for the standardization of the instrument. Two hundred five (205) children aged 3 to 5 years attending public daycare centers in the province of Mendoza were evaluated. The internal consistency of the test was estimated and item-subtest correlations were calculated. Additionally, an analysis of variance (ANOVA) with a priori contrast was used, T-scores per age group were calculated, and Cohen's $d$ was estimated to assess the magnitude of the differences found between the contrasted samples. The TEPSI displayed adequate psychometric properties as well as high sensitivity to assess age differences in children's psychomotor development. Argentine pre-school children showed better psychomotor performance when compared to their Chilean peers, in a context where both groups displayed different developmental rates. These results highlight the importance of developing updated local standards for the TEPSI that consider the socio-cultural features of each country, with a view to obtaining an accurate and reliable assessment of children's psychomotor development.
\end{abstract}

Keywords: scale, neurodevelopment, children, psychomotor, TEPSI
Para citar este artículo:

Espósito, A., Korzeniowski, C., \& Santini., M. (2018). Normas preliminares del Test de Desarrollo Psicomotor (TEPSI) para niños argentinos de 3 y 4 años. Liberabit, 24(1), 9-27. doi: 10.24265/liberabit.2018.v24n1.02
Este es un artículo Open Access bajo la licencia Creative Commons Atribución-NoComercial-CompartirIgual 4.0

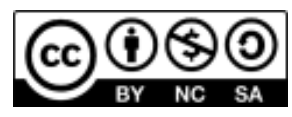




\section{Introducción}

Dentro del campo de la psicología que estudia el desarrollo infantil, ha existido gran interés por diseñar y aplicar pruebas de evaluación que permitan la detección temprana de alteraciones y/o trastornos en los años previos al ingreso a la escolaridad primaria. Esto resulta de fundamental importancia debido a los procesos de formación que van surgiendo en torno a la inteligencia y la personalidad de los niños a medida que transcurre su desarrollo.

Es ampliamente aceptado que la evaluación cognitiva y psicomotriz durante los primeros años de la infancia es un eslabón clave para la prevención y promoción de la salud de los niños (Calero, Carles, Mata, \& Navarro, 2010). La detección precoz de alteraciones en el neurodesarrollo permite realizar intervenciones oportunas, potenciando los procesos cognitivos a través de la implementación de actividades de estimulación. Asimismo, una evaluación diagnóstica precisa y minuciosa permite identificar variables que pueden estar afectando negativamente el desarrollo infantil, tales como condiciones de pobreza, falta de estimulación, bajas expectativas parentales, bajo nivel de instrucción de los progenitores, carencias nutricionales, hacinamiento, etc. (Canetti et al., 2002; Lipina, 2006; Mazzoni, Stelzer, Cervigni, \& Martino, 2014; Orozco-Hormaza, Sánchez-Ríos, \& CerchiaroCeballos, 2012; Schonhaut, Rojas, \& Kaempffer, 2003). En consecuencia, resulta fundamental detectar tempranamente problemas en el neurodesarrollo, debido a que sus alteraciones pueden repercutir negativamente en el aprendizaje, funcionamiento y adaptación del niño en edades posteriores (Flores, 2013).

En este sentido, detectar precozmente cualquier dificultad que pueda incidir de alguna manera en el desarrollo posterior del niño, se traducirá en mayores posibilidades de acción. En este contexto, los instrumentos de screening (tamizaje, despistaje, rastrillaje o cribado) adquieren una relevancia especial. A diferencia de las pruebas diagnósticas, este tipo de evaluaciones, de una manera rápida y con bajo costo, logra identificar determinada/s área/s en la/s que el niño evaluado presenta alguna desviación o trastorno (Merino-Soto, 2014a).

Lo expresado se fundamenta en el hecho de que las tareas de evaluación psicológica en la edad infantil cobran especial importancia, dado que muchos niños que no son identificados con dificultades antes del ingreso a la escolaridad primaria, posteriormente llegan a la consulta psicológica cuando la problemática ya está instalada, revistiendo un grado mayor de severidad que en edades más tempranas. Por ello, numerosos trabajos plantean la necesidad de instrumentar en esta población acciones de rastrillaje o screening con el fin de detectar precozmente casos clínicos que no se encuentren acudiendo a la consulta psicológica (CastroSolano \& Fernández-Liporace, 2017) o identificar niños con probable riesgo en una condición específica (Merino-Soto, 2014a).

Castro-Solano y Fernández-Liporace (2017) comentan que por lo general, este tipo de tareas de evaluación se realizan en ámbitos estatales o privados (establecimientos educativos y/o de salud), teniendo como objetivo identificar posibles trastornos psicológicos de aquellos sujetos que no han acudido a la consulta, permitiendo de esta manera, un filtrado de casos en riesgo. No obstante, una vez identificados deben derivarse posteriormente a una instancia de diagnóstico que permita la profundización y precisión de la evaluación.

En relación a ello, resulta de interés las recomendaciones vertidas por Meisels y Provence (1989), quienes sostienen que en las evaluaciones de screening o tamizaje se debe tener en cuenta, entre otros, los siguientes aspectos: a) el despistaje debe ser visto como un camino para una evaluación posterior en profundidad; b) debe ser aplicado en un ambiente controlado y con tareas relevantes al niño; c) el procedimiento debe ser confiable y válido; y d) debe tomar en cuenta las variaciones culturales. 
Otros autores sostienen que los tests de screening deben poseer una alta sensibilidad, de manera que permitan detectar a aquellos niños que presenten posibles indicadores de trastornos o psicopatología, no dejando fuera ningún caso probable, aunque luego en la fase de diagnóstico se confirme o no la presencia de estas dificultades (Castro-Solano \& Fernández-Liporace, 2017). Como contrapartida de esto, las pruebas de screening suelen registrar baja especificidad, ya que resulta imposible a partir de este tipo de instrumentos arribar a un diagnóstico diferencial del caso.

Específicamente dentro de la edad infantil, AnguloRamos y Merino-Soto (2012), plantean que es reducida la cantidad y variedad de pruebas en Latinoamérica que permiten evaluar eficazmente y de manera rápida el desarrollo psicomotor en niños menores de 6 años. Por ello, se torna imprescindible analizar las características psicométricas de los instrumentos existentes, como crear o adaptar instrumentos clínicamente sensibles y culturalmente relevantes en relación a las pruebas de screening.

En relación a lo expuesto, un aspecto de relevancia para arribar a una evaluación confiable, reside en la necesidad de contar con baremos locales y actualizados, de modo que cada niño pueda ser comparado con su verdadero grupo de referencia (Tornimbeni, Pérez, \& Olaz, 2008). En Argentina, instrumentos diseñados para tal fin son escasos y las validaciones de escalas foráneas han sido poco satisfactorias (Contini, 2013; Romero, 2011). Algunos instrumentos generados en nuestro país son la Escala Argentina de Inteligencia Sensorio-Motriz (EAIS) y la Prueba Nacional de Pesquisa (PRUNAPE), sin embargo, sus principales estudios se han realizado en Buenos Aires, desestimando las demás regiones del país (Lejarraga, 2008; Oiberman, Paolini, \& Mansilla, 2012).

Un aspecto que cobra relevancia en la elaboración de normas locales para la interpretación de un instrumento de evaluación se refiere a los aspectos culturales de cada grupo evaluado, fundamentalmente cuando los grupos pertenecen a países diferentes. Incluso se han hallado diferencias en los resultados de rendimiento en los tests cognitivos dentro de la misma cultura (diferencias intragrupos) (Merino-Soto, 2011). De hecho, cada vez con más énfasis se plantea la necesidad de considerar detenidamente los factores culturales cuando se adaptan o utilizan tests de un país a otro (Brenlla, 2004) o incluso de una región a otra dentro del mismo país.

En este sentido, estamos de acuerdo con lo señalado por Anastasi y Urbina (1998), al señalar que el comportamiento humano se encuentra influenciado por el medio sociocultural en el que el individuo se desarrolla y que los tests psicológicos son muestras de sus conductas, por lo que el efecto de la cultura debe reflejarse forzosamente en la ejecución del instrumento. En línea con lo expuesto, Butman et al. (2001), explican que las culturas son muy diferentes aún con el mismo idioma haciendo imprescindible una correcta traducción con adaptación de los instrumentos a las características locales.

Dentro de este marco, nuestro objeto de estudio es un instrumento de screening de origen chileno creado por Haeussler y Marchánt (1997), denominado Test de Desarrollo Psicomotor (TEPSI), el cual ha sido empleado en varios países de Latinoamérica (Vericat \& Orden, 2010), y tiene como propósito evaluar el desarrollo psicomotor en niños entre 2 y 5 años de edad. El mismo cuenta con un amplio reconocimiento en su país de origen, donde se ha utilizado en numerosos trabajos de investigación y a nivel de atención primaria en salud: «Chile Crece Contigo 2008-2011» (Atalah, Cordero, Guerra, Quezada, Carrasco, \& Romo, 2014). Se ha registrado el empleo y el estudio de este instrumento en países como Perú (Angulo-Ramos \& Merino-Soto, 2012; Astete et al., 2010; Merino-Soto, 2014a, Ministerio de Salud [MINSA], 2017), Ecuador (Jiménez-Capa, 2014), México (Myers \& Martínez, 2003) y Argentina, en el Programa de Seguimiento de Niños Prematuros del Hospital Materno Infantil Ramón Sardá 
(Gerometta, Aspres, Schapira, Granovsky, \& Vivas, 2014; Schapira, 2007; Schapira et al., 2008).

$\mathrm{Al}$ ser un instrumento de screening no provee información diagnóstica, sino solamente del estatus de «riesgo» del sujeto evaluado (Merino-Soto, 2014a), vale decir, permite detectar de manera gruesa o general riesgos o retrasos en el nivel de desarrollo del niño o en las áreas evaluadas (Haeussler \& Marchánt, 1997). Si bien el TEPSI cuenta con amplia aceptación, algunos autores han formulado diversas críticas a dicho instrumento. En este sentido, Angulo-Ramos y Merino-Soto (2012) comentan que si bien dicho test es útil y recomendado para hacer evaluaciones de rastrillaje temprano, es una herramienta que requiere más tiempo de lo justificado para realizar este tipo de evaluación. Dichos autores estiman un tiempo de administración entre 30 y 40 minutos el cual es variable según la disponibilidad conductual del niño. A este tiempo debe adicionarse el ordenamiento inicial y posterior de los materiales y el traslado del niño evaluado (Angulo-Ramos \& Merino-Soto, 2014). Por otra parte, la extensión del protocolo del test y las numerosas actividades propuestas, podrían asociarse con fluctuaciones en la atención del niño y fatiga, lo que repercutiría negativamente en su desempeño. Además, esta misma característica de la técnica requiere alto nivel de concentración de los evaluadores, en tanto deben mantenerse alerta para monitorear la conducta del niño, discernir entre una respuesta correcta o incorrecta, valorar inmediatamente el puntaje correspondiente y pasar a la siguiente tarea (Angulo-Ramos \& Merino-Soto, 2012, 2014).

Lo expuesto es consistente con lo planteado por Castro-Solano y Fernández-Liporace (2017), quienes señalan que estos tipos de pruebas deben ser breves, fáciles de administrar y evaluar, de manera que permitan examinar a una gran cantidad de sujetos en el menor tiempo posible. Esto permitiría detectar precozmente los casos positivos potenciales y derivarlos a la instancia diagnóstica y su eventual tratamiento. Estos autores destacan que estos tipos de pruebas, por su alta sensibilidad, hacen que las puntuaciones de los sujetos que se encuentran en riesgo se eleven muy fácilmente aun cuando estos no manifiesten gran cantidad de síntomas. En esta línea, otros autores comentan que la interpretación que se le otorga a los puntajes obtenidos por el niño en la prueba (normal, riesgo y retraso) genera etiquetas diagnósticas que podrían influir negativamente en un examinador poco entrenado, ya que puede considerar que no es necesaria una segunda evaluación en profundidad dado que el niño ha sido identificado con «retraso», evitando con ello que el menor sea evaluado por otros instrumentos más precisos (AnguloRamos \& Merino-Soto, 2012).

Por otro lado, algunos estudios han señalado que el subtest de lenguaje del TEPSI no sería tan preciso a la hora de discriminar entre niños con y sin problemas fonológicos o de lectoescritura (HeresiVenegas \& Avaria-Benaprés, 2014; Schonhaut, Maggiolo, Barbieri, Rojas, \& Salgado, 2007). No obstante, vale aclarar que el TEPSI mantiene una gran vigencia de uso en la actualidad, y si bien podría desaconsejarse su aplicación masiva para una evaluación rápida de screening, consideramos que el empleo de este instrumento es altamente sugerido en contextos donde no se requieran evaluaciones de un gran número de niños, con un ambiente cómodo y apropiado para trabajar durante un lapso de tiempo moderado con niños preescolares y a través de un evaluador correctamente entrenado.

En función de lo expuesto, el presente trabajo pretende contribuir a la evaluación neuropsicológica de niños argentinos en edad preescolar a través de la obtención de baremos preliminares del TEPSI. Los objetivos específicos del trabajo son: 1) describir el nivel de desarrollo psicomotor, en las áreas de coordinación, motricidad y lenguaje, en niños mendocinos de 3 a 5 años de edad y analizar si el mismo varía en función de la edad y género de los niños participantes; 2) contrastar el nivel de desarrollo psicomotor y su velocidad de crecimiento entre la muestra argentina y la muestra chilena empleada para 
la estandarización del TEPSI, y 3) elaborar normas locales preliminares del TEPSI para preescolares argentinos.

\section{Método}

\section{Participantes}

Se utilizó una muestra no probabilística intencional (Hernández, Fernández, \& Baptista, 2006), conformada por 205 niños, de 3 a 5 años de edad: 103 mujeres y 102 varones, que asistían a jardines maternales municipales de diferentes departamentos (distritos) de la provincia de Mendoza. Los establecimientos educativos están insertos en contextos urbanos, la población que asiste a los mismos vive en áreas cercanas a los jardines y se caracteriza por pertenecer a un nivel socioeconómico medio y en algunos casos, medio-bajo. Si bien, no se administró un instrumento para medir los aspectos sociodemográficos de la muestra en estudio, a través de una entrevista con los docentes se obtuvo información suficiente para una caracterización de la misma. Así, se observó que el nivel educativo de la mayoría de los padres y madres de los niños participantes oscilaba entre primaria y secundaria completa. En relación a la ocupación, en las madres variaba entre ama de casa, empleada del municipio, empleada administrativa o de comercio y docentes; y en relación a los padres, la mayoría eran empleados municipales, administrativos o trabajaban de manera independiente.
Los jardines maternales reciben subsidios económicos del estado para su funcionamiento, debido a que los padres de los niños que concurren no aportan una cuota mensual durante el ciclo lectivo, pero sí los materiales de trabajo que sus hijos emplearán durante el año (hojas, colores, etc.). El personal docente recibe su remuneración por parte del municipio. Los juegos didácticos del jardín pertenecen a la institución y esta les brinda a los niños los alimentos para el desayuno, las meriendas y/o el almuerzo. Todos los jardines funcionan desde la mañana hasta última hora de la tarde y su plantel docente está conformado por una directora, una coordinadora, docentes de nivel inicial, y auxiliares de jardines maternales. Respecto a su estructura edilicia, todos los jardines son de una sola planta, con un espacio recreativo al aire libre, un espacio para el directivo y diferentes salas a donde concurren los niños agrupados por edad. Cada sala está bajo la responsabilidad de una docente con título de nivel inicial para trabajar con el grupo de niños.

En relación a los participantes que conformaron la muestra se empleó como criterios de exclusión que los infantes no estuviesen realizando ningún tratamiento psicológico/psiquiátrico, y que no presentaran problemas sensoriales, intelectuales o trastornos del desarrollo previamente diagnosticados. La muestra fue dividida en cuatro grupos en función de la edad (ver Tabla 1).

Tabla 1

Composición sociodemográfica de la muestra

\begin{tabular}{clccc}
\hline Grupo & \multicolumn{1}{c}{ Rango etario } & $n$ & niñas & niños \\
\hline 1 & 3 años a 3 años, 6 meses & 68 & 38 & 30 \\
2 & 3 años, 7 meses a 4 años & 86 & 46 & 40 \\
3 & 4 años, 1 mes a 4 años, 6 meses & 30 & 13 & 17 \\
4 & 4 años, 7 meses a 5 años, 1 mes & 17 & 6 & 11 \\
\hline
\end{tabular}




\section{Diseño}

El enfoque de este estudio es cuantitativo; el alcance de la investigación es descriptivo con un diseño no experimental y transversal (Hernández, Fernández, \& Baptista, 2006).

\section{Instrumento}

Test de Desarrollo Psicomotor (TEPSI, Haeussler \& Marchánt, 1997). Este instrumento es una técnica de screening que permite evaluar el desarrollo psicomotor en niños entre 2 y 5 años de edad. Está compuesto de 52 ítems o tareas organizadas en tres subtests: Coordinación, Lenguaje y Motricidad. A su vez, permite obtener un puntaje total que valora el nivel general de desarrollo psicomotor. El subtest de Coordinación evalúa en 16 ítems la habilidad del niño para manipular objetos y dibujar, a través de conductas como construir torres con cubos, enhebrar una aguja, reconocer y copiar figuras geométricas. El subtest de Lenguaje evalúa en 24 ítems aspectos de comprensión y expresión del lenguaje a través de tareas como nombrar objetos, definir palabras, verbalizar acciones. El subtest de Motricidad evalúa en 12 ítems la habilidad del niño para controlar su propio cuerpo a través de conductas como saltar en un pie, tomar una pelota, caminar en punta de pie. El test se administra de manera individual en un lapso promedio de $30 \mathrm{a}$ 40 minutos. Cada respuesta se puntúa con 0 o 1, según sea correcta o incorrecta respectivamente, obteniéndose el puntaje de los tres subtests y el puntaje total mediante sumatoria simple. Se dispone de baremos para población chilena por grupo etario y género, los cuales permiten determinar desarrollo normal, retraso o riesgo (Haeussler \& Marchánt, 1997).

En lo que respecta a los niveles de confiabilidad del instrumento, los índices de consistencia interna resultaron satisfactorios para el puntaje total del TEPSI $(K-R 20=.94)$, así como para cada subescala: Coordinación $(K-R 20=.89)$, Lenguaje $(K-R 20=$ $.94)$ y Motricidad $(K-R 20=.82)$ (Haeussler \& Marchánt, 1997). Asimismo, la confiabilidad inter- examinador para el puntaje total del TEPSI $(r=.98)$ y para las tres subescalas (Coordinación: $r=.93$, Lenguaje: $r=.97$ y Motricidad $r=.97$ ) resultó excelente (Haeussler \& Marchánt, 1997).

En la muestra argentina en estudio se obtuvieron adecuados índices de consistencia interna para el puntaje total del TEPSI $(K-R 20=.91)$ y para las tres subescalas que conforman el test: Coordinación $(K-R 20=.75)$, Lenguaje $(K-R 20=.86)$ y Motricidad $(K-R 20=.71)$. Estos valores resultan apropiados para una prueba de tamizaje teniendo en cuenta que las tareas propuestas son heterogéneas (Angulo-Ramos \& Merino-Soto, 2012). Por otra parte, se exploró la validez del constructo a través de la estimación de la relación entre los ítems y su factor. Se observó que el $90 \%$ de los ítems que conforman el TEPSI presentaron una adecuada relación con su factor, 40 ítems presentaron correlaciones entre .40 y .73, y 7 ítems arrojaron correlaciones entre .25 y .39. El 10\% restante de los ítems (ítems 3, 6 y 7 de Coordinación e ítems 3 y 6 de Motricidad), no se correlacionó con su factor, lo que podría señalar que estos reactivos presentan dificultades para medir aspectos del constructo para el cual fueron diseñados. Estos resultados no son concordantes con los obtenidos en la muestra chilena de estandarización del TEPSI, a excepción del ítem 7 del factor Coordinación el cual exhibió una correlación débil con su factor (Haeussler \& Marchánt, 1997). Estudios posteriores deberían continuar examinando las propiedades psicométricas de estos indicadores y su variabilidad en función del contexto sociocultural.

\section{Procedimiento}

En primera instancia, se procedió a contactar a los directores de los jardines maternales municipales participantes, a fin de obtener su autorización para desarrollar el proyecto. Luego, se realizó una reunión informativa con los padres de los niños. Se incluyeron en el estudio a aquellos infantes cuyos padres hubieran firmado el consentimiento informado, en el cual quedaron explicitados los términos de 
confidencialidad, la participación libre y voluntaria, los objetivos de investigación, la metodología de trabajo y la entrega de informes de devolución. Luego, se administró a los niños, de manera individual, el TEPSI en una o dos jornadas de trabajo. Vale decir que a los niños que mostraban interés en trabajar, motivación en la realización del test y no expresaban signos de cansancio y/o aburrimiento se les administraba la técnica en una sola sesión de 40 o 45 minutos aproximadamente. Aquellos niños que presentaban signos de cansancio y/o aburrimiento, la técnica se les administraba en dos días diferentes, en un día se evaluaba el área de Lenguaje por su extensión y en la otra jornada los subtests de Motricidad y Coordinación. La mayoría de los niños pudieron realizar el test en una sola jornada de trabajo debido al interés de estos por trabajar con los evaluadores. Casos excepcionales fueron evaluados en dos sesiones como, por ejemplo, niños que mostraban excesiva inquietud motora o distraibilidad.

La administración del TEPSI a la totalidad de la muestra se llevó a cabo en el lapso de un ciclo lectivo, para lo cual se entrenó a alumnos avanzados de la licenciatura en Psicología. Dicho entrenamiento estuvo a cargo de la primera autora de este estudio, conformando de esta manera un grupo de 8 evaluadores capacitados en la administración y puntuación del test bajo los lineamientos pertinentes a la evaluación neuropsicológica con niños de edad preescolar.

\section{Análisis de datos}

Se condujeron una serie de procedimientos con el fin de preparar los datos para los análisis propuestos. En primer lugar, se evaluó el patrón de valores perdidos para estimar si el mismo respondía a una distribución aleatoria mediante la rutina de Análisis de los Valores Perdidos del SPSS 19. En un segundo paso, se identificaron casos atípicos univariados mediante la inspección gráfica de los datos (boxplots).
En un tercer paso, se realizó una estimación directa de la normalidad estadística de las distribuciones de los puntajes mediante el test de Shapiro Wilks, el cual se seleccionó debido a que ha demostrado ser un test robusto, aplicable en todo tipo de distribución y tamaño muestral (Razali \& Wah, 2011). Dicho análisis se complementó con la valoración de los índices de asimetría y curtosis en cada variable. Como criterio para evaluar los índices de asimetría y curtosis se consideró como adecuados valores inferiores a +2 y -2 (George \& Mallery, 2011).

Para describir el nivel de desarrollo psicomotor en los niños participantes, se calcularon los estadísticos descriptivos de Coordinación, Motricidad, Lenguaje y puntaje total del TEPSI para cada grupo etario. Luego, se realizó un análisis de varianza multivariado (MANOVA) para valorar si el desempeño psicomotor de los niños fluctuaba según género y edad. Siguiendo a Merino-Soto (2014b), se decidió complementar este análisis con un ANOVA con contraste a priori a fin de valorar la direccionalidad del efecto de la edad en el desarrollo psicomotor infantil.

Posteriormente, en miras a alcanzar el segundo objetivo del estudio, se contrastaron los resultados obtenidos en la muestra argentina con la muestra chilena de estandarización del TEPSI a fin de estimar posibles diferencias en el desempeño psicomotriz y en el ritmo de crecimiento en función del contexto sociocultural. Para este último estudio, se calculó la $d$ de Cohen con sus intervalos de confianza (Cohen, 1992; Coe \& Merino-Soto, 2003; Merino-Soto, 2014b) a fin de valorar la magnitud de las diferencias en el desempeño del TEPSI.

Finalmente, para dar respuesta al tercer objetivo del trabajo se elaboraron baremos preliminares del TEPSI para preescolares mendocinos, para lo cual se estimó la distribución percentilar y las puntuaciones típicas (T) de los puntajes directos de los tres factores y el puntaje total del test en cada grupo etario. 


\section{Resultados}

No se detectaron casos perdidos. La inspección gráfica de los datos permitió identificar cuatro casos atípicos. Previo a la exclusión de estos casos, se examinaron los protocolos de respuesta de los niños. Atendiendo a las observaciones registradas en los mismos, en las cuales se dejaba constancia de conductas de fatiga o desmotivación en los preescolares y a que los niños presentaron un rendimiento irregular en el test, las autoras del trabajo decidieron no incluir estos cuatro casos en el presente trabajo. En consecuencia, la muestra final quedó conformada por 201 niños.

Se estimó la normalidad de la distribución de las variables para la muestra en estudio. Los resultados obtenidos mediante el test Shapiro Wilks indicaron que las variables Coordinación, Motricidad, Lenguaje y puntaje total del TEPSI no presentaron una distribución normal en la mayoría de los grupos en

estudio (ver Tabla 2). Sin embargo, los índices de asimetría y curtosis evidenciaron una alta semejanza de las distribuciones de las variables con la curva normal, registrándose valores entre +1 y -1 en todas las variables en los cuatros grupos etarios, a excepción de Lenguaje la cual arrojó valores entre +2 y -2 en el grupo 3 y 4 . Estos valores son considerados adecuados para conducir análisis paramétricos. Asimismo, la literatura informa que si los datos son simétricos, los análisis de varianza resultan robustos a las desviaciones de la normalidad (Angulo-Ramos \& Merino-Soto, 2014; George \& Mallery, 2001).

\section{Estudio descriptivo y análisis multivariado}

Para dar respuesta al primer objetivo del trabajo, se calcularon los valores promedio y las desviaciones típicas de las variables Coordinación, Motricidad, Lenguaje y Nivel de desarrollo psicomotriz (puntaje total del TEPSI) para cada grupo etario (ver Tabla 2).

Tabla 2

Estadísticos descriptivos del TEPSI para niños mendocinos según edad

\begin{tabular}{|c|c|c|c|c|c|c|c|c|c|c|c|c|c|}
\hline & \multirow[b]{2}{*}{$n$} & \multicolumn{3}{|c|}{ Coordinación } & \multicolumn{3}{|c|}{ Lenguaje } & \multicolumn{3}{|c|}{ Motricidad } & \multicolumn{3}{|c|}{ Total } \\
\hline & & $M$ & $D E$ & $W$ & $M$ & $D E$ & $W$ & $M$ & $D E$ & $W$ & $M$ & $D E$ & $W$ \\
\hline G1 & 68 & 8.47 & 2.32 & .017 & 13.51 & 5.85 & .275 & 7.53 & 2.40 & .120 & 29.66 & 8.93 & .316 \\
\hline G2 & 86 & 9.58 & 2.24 & .027 & 17.07 & 4.87 & $<.001$ & 8.69 & 2.24 & .001 & 35.42 & 7.80 & .002 \\
\hline G3 & 30 & 11.33 & 2.40 & .205 & 19.73 & 3.02 & .001 & 9.83 & 1.64 & .013 & 40.90 & 5.41 & .065 \\
\hline G4 & 17 & 13.06 & 1.52 & .266 & 22.18 & 1.98 & .003 & 11.06 & .97 & .007 & 46.06 & 3.31 & .452 \\
\hline
\end{tabular}

Nota: $W=$ Shapiro Wilks.

Posteriormente, se condujo un MANOVA a fin de valorar si el nivel de desarrollo psicomotor fluctuaba según la edad y el género de los niños. Los resultados obtenidos señalaron que la coordinación psicomotriz, la motricidad, el lenguaje y el nivel general del desarrollo psicomotor de los niños variaron en función de la edad $\left(\lambda=7.48, p<.001, \eta^{2}=.13\right)$. En relación con la variable género no se hallaron diferencias en ninguna de las cuatro variables contrastadas $(\lambda=.84$, $\left.p<.50, \eta^{2}=.017\right)$. Asimismo, no se registraron diferencias en el desarrollo psicomotriz de los niños en función de la interacción Edad x Género $(\lambda=51$, $p<.91, \eta^{2}=.01$ ).

A fin de estudiar la direccionalidad del efecto de la edad sobre el desarrollo psicomotor en los niños, se decidió realizar un ANOVA con contraste $a$ priori (ver Tabla 3). Los resultados obtenidos permitieron observar que el contraste lineal a priori pudo explicar entre el 98\% y el $99.9 \%$ de la variabilidad del desempeño psicomotor en los niños, mostrando mayor fuerza explicativa que el ANOVA general (ver 
Tabla 3). Los datos obtenidos señalaron que a medida que los niños aumentaban en edad, mejoraban su desempeño en coordinación $\left(F=73.09, p<.001, \eta^{2}\right.$ $=.28)$, lenguaje $\left(F=62.43, p<.001, \eta^{2}=.24\right)$, motricidad $\left(F=49.01, p<.001, \eta^{2}=.20\right) \mathrm{y}$, en consecuencia, en el nivel general de desarrollo psicomotriz $\left(F=86.44, p<.001, \eta^{2}=.30\right)$.

Tabla 3

Efecto de la edad sobre el desarrollo psicomotor

\begin{tabular}{cccccccc}
\hline Variable & Fuente & $S C$ & $g l$ & $M C$ & $F$ & $p$ & $\eta^{2}$ \\
\hline Coordinación & Entre grupos & 375.06 & 3 & 125.02 & 24.84 & $<.001$ & .27 \\
& Contraste & 367.83 & 1 & 367.83 & 73.09 & $<.001$ & .28 \\
& No contraste & 7.22 & 2 & 3.61 & .71 & .489 & .01 \\
& Intra grupo (error) & 991.48 & 197 & 5.03 & & & \\
\hline \multirow{2}{*}{ Lenguaje } & Entre grupos & 1487.58 & 3 & 495.86 & 21.06 & $<.001$ & .24 \\
& Contraste & 1469.53 & 1 & 1469.53 & 62.43 & $<.001$ & .24 \\
& No contraste & 18.05 & 2 & 9.03 & .38 & .682 & .00 \\
& Intra grupo (error) & 4636.90 & 197 & 23.54 & & & \\
\hline \multirow{2}{*}{ Motricidad } & Entre grupos & 226.09 & 3 & 75.36 & 16.34 & $<.001$ & .20 \\
& Contraste & 226.05 & 1 & 226.05 & 49.01 & $<.001$ & .20 \\
& No contraste & .04 & 2 & .02 & .005 & .996 & .00 \\
& Intra grupo (error) & 908.57 & 197 & & & & \\
\hline \multirow{2}{*}{ TEPSI TOTAL } & Entre grupos & 5069.02 & 3 & 1689.67 & 28.83 & $<.001$ & .30 \\
& Contraste & 5065.33 & 1 & 5065.33 & 86.44 & $<.001$ & .30 \\
& No contraste & 3.69 & 2 & 1.85 & .03 & .969 & .00 \\
& Intra grupo (error) & 11543.79 & 197 & & & & \\
\hline
\end{tabular}

Nota: $\mathrm{SC}=$ suma de cuadraros; MC = media cuadrática; $\eta^{2}$ = magnitud del efecto.

\section{Estudio comparativo}

Se compararon los datos obtenidos en la muestra argentina con los baremos realizados en Chile a fin de identificar variabilidad en el desarrollo psicomotor de los infantes según el país de origen.

La Tabla 4 refleja que los niños mendocinos presentaron puntajes promedios superiores que sus pares chilenos en los tres subtests que conforman el TEPSI y en el puntaje total, en todos los rangos etarios examinados. Analizando la magnitud de las diferencias se observaron diferencias de moderadas a grandes en Motricidad ( $d=.46$ a .93 ) y en el puntaje total de TEPSI ( $d=.23$ a .91$)$ a favor de los preescolares mendocinos.
En Coordinación, las diferencias oscilaron de pequeñas a moderadas ( $d=.24$ a .39), siendo la excepción el grupo de 3 años y medio a 4 años (G2) en el cual los niños de ambos países mostraron un desempeño similar $(d=.07$, IC $=-.22$, .36). En Lenguaje, se registró un desempeño semejante entre los niños argentinos y chilenos a excepción del grupo etario mayor, en el cual se observaron marcadas diferencias a favor de los primeros $(d=.85$, IC $=$ $.33,1.37$ ). En general, las magnitudes halladas indican diferencias marcadas entre los puntajes promedio en Motricidad, puntaje total del TEPSI y Coordinación entre mendocinos y chilenos. 
Tabla 4

Diferencias en el nivel de desarrollo psicomotor entre mendocinos y chilenos.

\begin{tabular}{|c|c|c|c|c|c|c|c|c|c|c|c|c|c|}
\hline & \multirow{2}{*}{ País } & \multicolumn{3}{|r|}{ Coordinación } & \multicolumn{3}{|c|}{ Lenguaje } & \multicolumn{3}{|c|}{ Motricidad } & \multicolumn{3}{|c|}{ Total } \\
\hline & & $n$ & $M$ & $D E \quad d($ IC95\%) & $M$ & $D E$ & $d$ (IC95\%) & $M$ & $D E$ & $d$ (IC95\%) & $M$ & $D E$ & $d(\mathrm{IC} 95 \%)$ \\
\hline G1 & $\begin{array}{l}\text { Mza } \\
\text { Ch }\end{array}$ & $\begin{array}{l}68 \\
90\end{array}$ & $\begin{array}{l}8.47 \\
7.53\end{array}$ & $\begin{array}{l}2.32 \\
2.54\end{array} .39(.08 .70)$ & $\begin{array}{l}13.51 \\
13.14\end{array}$ & $\begin{array}{l}5.85 \\
5.27\end{array}$ & $.02(-.11 .51)$ & $\begin{array}{l}7.53 \\
6.09\end{array}$ & $\begin{array}{l}2.40 \\
2.06\end{array}$ & $.64(.32 .95)$ & $\begin{array}{l}29.66 \\
26.76\end{array}$ & $\begin{array}{l}8.93 \\
8.29\end{array}$ & $.34(.02 .65)$ \\
\hline G2 & $\begin{array}{c}\text { Mza } \\
\text { Ch }\end{array}$ & $\begin{array}{l}86 \\
90\end{array}$ & $\begin{array}{l}9.58 \\
9.42\end{array}$ & 2.24 .07 (-.22 .36) & $\begin{array}{l}17.07 \\
16.63\end{array}$ & $\begin{array}{l}4.87 \\
4.23\end{array}$ & $.10(-.19 .39)$ & $\begin{array}{l}8.69 \\
7.69\end{array}$ & $\begin{array}{l}2.24 \\
2.09\end{array}$ & $.46(.16 .75)$ & $\begin{array}{l}35.42 \\
33.74\end{array}$ & $\begin{array}{l}7.80 \\
6.62\end{array}$ & $.23(-.06 .53)$ \\
\hline G3 & $\begin{array}{c}\text { Mza } \\
\text { Ch }\end{array}$ & $\begin{array}{l}30 \\
90\end{array}$ & $\begin{array}{l}11.33 \\
10.80\end{array}$ & $\begin{array}{l}2.40 \\
1.92\end{array} .24(-.17 .65)$ & $\begin{array}{l}19.73 \\
18.32\end{array}$ & $\begin{array}{l}3.02 \\
3.31\end{array}$ & $.44(.03 .85)$ & $\begin{array}{l}9.83 \\
8.44\end{array}$ & $\begin{array}{l}1.64 \\
1.86\end{array}$ & $.79(.361 .22)$ & $\begin{array}{l}40.90 \\
37.57\end{array}$ & $\begin{array}{l}5.41 \\
5.16\end{array}$ & $.63(.21-1.04)$ \\
\hline G4 & $\begin{array}{c}\text { Mza } \\
\text { Ch }\end{array}$ & $\begin{array}{l}17 \\
90\end{array}$ & $\begin{array}{l}13.06 \\
12.48\end{array}$ & $\begin{array}{l}1.52 \\
1.99\end{array} .33(-.19 .85)$ & $\begin{array}{l}22.18 \\
19.93\end{array}$ & $\begin{array}{l}1.98 \\
3.18\end{array}$ & $.85(.331 .37)$ & $\begin{array}{c}10.82 \\
9.51\end{array}$ & $\begin{array}{c}.97 \\
1.73\end{array}$ & $.93(.661 .20)$ & $\begin{array}{l}46.06 \\
41.92\end{array}$ & $\begin{array}{l}3.31 \\
5.50\end{array}$ & $.91(.391 .43)$ \\
\hline
\end{tabular}

Nota: $d=$ magnitud del efecto

Por otra parte, para estimar la velocidad de los cambios en el desarrollo se calculó la diferencia entre los puntajes promedios en cada una de las variables, en los diferentes rangos de edad (M2-M1, M3-M2, M4-M3), en la muestra mendocina y chilena. Los resultados obtenidos indicaron patrones similares de crecimiento en Coordinación y Motricidad entre los dos grupos, registrándose un incremento del puntaje promedio en dichas variables, entre 1 y 1.5 , cada seis meses de edad. En Lenguaje se registraron diferencias en el patrón de desarrollo: los niños mendocinos mostraron un incremento entre 3.5 a 2.5 puntos cada seis meses de edad, en cambio, sus pares chilenos mostraron una ganancia de 3.5 puntos entre los 3 años y los 3 años y medio, y posteriormente, presentaron una ganancia de 1.5 cada seis meses. Estos resultados podrían indicar que los niños mendocinos y chilenos aumentaron de manera similar sus competencias lingüísticas entre los 3 años y los 3 años y medio, aunque luego los niños mendocinos exhibieron una velocidad de crecimiento mayor que sus pares chilenos. Similarmente, se registraron diferencias en el incremento del puntaje total del TEPSI, los niños mendocinos aumentaron en promedio 5.5 puntos cada seis meses, en cambio, los chilenos mostraron un crecimiento más irregular, aumentando siete puntos entre los 3 años y 3 años y medio, cinco puntos entre los 3 años y medio y los 4 años, y cuatro puntos entre los 4 años y medio y los 5 años de edad.

\section{Elaboración de normas preliminares del TEPSI para preescolares mendocinos}

En relación al tercer objetivo, el cual apunta a la elaboración de normas preliminares locales del TEPSI para población mendocina, a continuación, en la Tabla 5 se exponen los baremos correspondientes a los cuatros grupos etarios.

Una vez obtenidas las puntuaciones típicas $(\mathrm{T})$ de la muestra, pudo estimarse para el total de los niños participantes $(n=201)$ el porcentaje que presentó un desarrollo esperable (puntaje $\mathrm{T}>40$ ), retraso (puntaje $\mathrm{T}<30$ ) o riesgo (puntaje $\mathrm{T}$ entre 30 y 40 ) en cada una de las variables estudiadas. En relación al porcentaje de niños que obtuvieron desempeño normal, en riesgo o con retraso en el TEPSI, Coordinación fue la variable en la cual los niños participantes presentaron mayores niveles de dificultad (riesgo $18.9 \%$ y retraso $3.5 \%$ ). Sin embargo, los porcentajes de niños por debajo y por encima de los valores esperados, son similares en las cuatro variables del TEPSI, observándose que aproximadamente el $80 \%$ de los casos alcanzaron puntajes esperados para su edad, el 15\% presentó riesgo y, finalmente, los niños que perfilan en el grupo de retraso, no superaron el 5\% (ver Anexo). 
Tabla 5

Normas preliminares del TEPSI para preescolares mendocinos por edad

\begin{tabular}{|c|c|c|c|c|c|c|c|c|c|c|c|}
\hline \multicolumn{6}{|c|}{3 años a 3 años, 6 meses } & \multicolumn{6}{|c|}{3 años, 7 meses a 4 años } \\
\hline $\mathrm{C}$ & $\mathrm{L}$ & $\mathrm{M}$ & DP & Pc & $\mathrm{T}$ & $\mathrm{C}$ & $\mathrm{L}$ & $\mathrm{M}$ & DP & Pc & $\mathrm{T}$ \\
\hline$>13.00$ & $>24.00$ & $>12.00$ & $>49.00$ & $>99$ & 80 & $>14.00$ & $>24.00$ & $>12.00$ & $>48.00$ & $>99$ & 80 \\
\hline 13.00 & 24.00 & 12.00 & 47.86 & 98 & 70 & 14.00 & 24.00 & 12.00 & 46.52 & 98 & 70 \\
\hline 10.96 & 20.00 & 10.00 & 39.96 & 84 & 60 & 12.00 & 22.00 & 11.00 & 44.00 & 84 & 60 \\
\hline 8.50 & 13.00 & 7.50 & 29.00 & 50 & 50 & 10.00 & 18.00 & 9.00 & 38.00 & 50 & 50 \\
\hline 6.00 & 7.00 & 5.00 & 19.04 & 16 & 40 & 7.00 & 11.00 & 6.00 & 26.00 & 16 & 40 \\
\hline 4.38 & .76 & 2.38 & 12.38 & 2 & 30 & 5.00 & 5.74 & 3.00 & 17.70 & 2 & 30 \\
\hline$<4.00$ & $<.76$ & $<2.00$ & $<12.00$ & $<1$ & 20 & $<5.00$ & $<5.00$ & $<3.00$ & $<14.00$ & $<1$ & 20 \\
\hline \multicolumn{6}{|c|}{4 años, 1 mes a 4 años, 6 meses } & \multicolumn{6}{|c|}{4 años, 7 meses a 5 años, 1 mes } \\
\hline $\mathrm{C}$ & $\mathrm{L}$ & $\mathrm{M}$ & DP & Pc & $\mathrm{T}$ & $\mathrm{C}$ & $\mathrm{L}$ & $\mathrm{M}$ & DP & Pc & $\mathrm{T}$ \\
\hline$>15.00$ & $>23.00$ & $>12.00$ & $>49.00$ & $>99$ & 80 & $>16.00$ & $>24.00$ & $>12.00$ & $>51.00$ & $>99$ & 80 \\
\hline 15.00 & 23.00 & 12.00 & 49.00 & 98 & 70 & 16.00 & 24.00 & 12.00 & 51.00 & 98 & 70 \\
\hline 14.00 & 22.04 & 12.00 & 48.00 & 84 & 60 & 15.00 & 24.00 & 12.00 & 49.12 & 84 & 60 \\
\hline 11.00 & 20.50 & 10.00 & 40.00 & 50 & 50 & 13.00 & 23.00 & 11.00 & 46.00 & 50 & 50 \\
\hline 9.00 & 16.96 & 7.96 & 36.84 & 16 & 40 & 11.00 & 19.88 & 9.88 & 41.88 & 16 & 40 \\
\hline 5.00 & 12.00 & 7.00 & 27.00 & 2 & 30 & 11.00 & 17.00 & 7.00 & 39.00 & 2 & 30 \\
\hline$<5.00$ & $<12.00$ & $<7.00$ & $<27.00$ & $<1$ & 20 & $<11.00$ & $<17.00$ & $<7.00$ & $<39.00$ & $<1$ & 20 \\
\hline
\end{tabular}

Nota: $\mathrm{C}=$ coordinación, $\mathrm{L}=$ lenguaje; $\mathrm{M}=$ motricidad.; $\mathrm{DP}=$ Desarrollo Psicomotor; $P c=$ Percentiles; $\mathrm{T}=$ puntuación T.

\section{Discusión}

El presente trabajo brinda nuevos datos sobre el uso y las propiedades psicométricas del Test de Desarrollo Psicomotor TEPSI en preescolares argentinos.

Los resultados obtenidos, en concordancia con otros previos (Haeussler \& Marchánt, 1997; JiménezCapa, 2014), señalaron que el TEPSI es una herramienta confiable y válida para valorar el desarrollo psicomotor en niños argentinos de 3 a 5 años de edad. Como instrumento de tamizaje, permite identificar niños con dificultades en el control motor de su cuerpo, en coordinación psicomotriz y en la comprensión y/o expresión del lenguaje. A partir de esta evaluación inicial resulta posible delinear un diagnóstico en profundidad con miras de implementar intervenciones tempranas. En consecuencia, el TEPSI es un valioso instrumento de screening que puede colaborar con la identificación precoz de alteraciones en el neurodesarrollo infantil.
Por otro lado, el test mostró alta sensibilidad para valorar los cambios en el desarrollo cognitivo y psicomotor de los niños conforme aumentan en edad. Estos resultados han sido registrados en estudios previos (Angulo-Ramos \& Merino-Soto, 2012; Haeussler \& Marchánt, 1997; Jiménez-Capa, 2014) y resultan coincidentes con la literatura, donde se especifica que a medida que los niños crecen, van adquiriendo mayores habilidades y más experiencia, lo que les permitiría ir alcanzando más logros y avances en sus habilidades. En consecuencia, a medida que los niños avanzan en edad son más capaces de responder con éxito a los ítems del test debido al mayor nivel de desarrollo alcanzado, mayor maduración y mayor capacidad para realizar aprendizajes complejos (Papalia, Wendkos-Olds, \& Feldman, 2009; Portellano, 2007). 
En cuanto a las diferencias de género, son numerosos los estudios que destacan que las mujeres tienden a presentar mejor rendimiento que los varones en las tareas propuestas por las escalas de neurodesarrollo en la edad preescolar (Canetti et al., 2002; Lejarraga 2008; Otárola-Suárez, 2012; Papalia et al., 2009; Schonhaut et al., 2007), aunque se ha visto que esta situación no obedece a diferencias a nivel de la inteligencia (Garaigordobil \& Amigo, 2010). En el presente trabajo, contrariamente a lo reseñado, no se observaron diferencias entre niños y niñas.

La ausencia de diferencias de género en el desarrollo psicomotor puede asociarse a dos explicaciones tentativas. Por una parte, los avances en el área del cuidado pre y perinatal han provocado una disminución de casos de niños con problemas al nacer, los cuales afectaban mayormente a los varones (Cova, Valdivia, \& Maganto, 2005). Desde otra vertiente, se podría presumir que en este último tiempo se han ido produciendo cambios culturales que han influenciado los estilos de crianza, inclinando a los cuidadores a aplicar estilos más similares entre varones y mujeres, a diferencia de las pautas de crianza tradicionales (Cova et al., 2005).

Por otra parte, los resultados del estudio indicaron que el $83.5 \%$ de los niños presentaron un desarrollo psicomotor esperable para su edad, mientras que el $14.5 \%$ se ubicó en la franja de riesgo y el 2\% presentó retraso. Estos porcentajes son semejantes a los obtenidos en estudios previos realizados en Argentina y en otros países de América del Sur, en los cuales se indica que entre el $68 \%$ y $85 \%$ de los niños alcanza niveles de desarrollo psicomotor de acuerdo a la norma y entre el $15 \%$ y $26 \%$ se ubica en las categorías de riesgo y retraso (Atalah et al., 2014; Canetti et al., 2002; Gerometta et al., 2014; Heresi-Venegas \& Avaria-Benaprés, 2014; Lejarraga, 2008; Oiberman et al., 2012; Schapira et al., 2008; Schonhaut et al., 2003).

En relación a la distribución de los niños por área, el mayor porcentaje de niños en riesgo y retraso se presentó en coordinación psicomotriz, a diferencia de estudios previos que refieren mayores dificultades en el área del lenguaje. Numerosas investigaciones concluyen que la función del lenguaje suele ser la más comprometida en el desarrollo infantil, registrando los mayores porcentajes de riesgo y retraso en comparación con los otros indicadores del TEPSI (Aguinaga-Espinoza, 2012; Atalah et al., 2014; Canetti et al., 2002; Lipina, 2006; Otárola-Suárez, 2012; Schonhaut et al., 2003; 2007). Sin embargo, el presente trabajo no aportó evidencia en este sentido.

Como posible explicación a estos resultados disidentes es preciso actualizar que el subtest Lenguaje ha sido objeto de críticas y observaciones. Algunos expertos señalan que el mismo mide aspectos demasiado básicos y cuantitativos del lenguaje, brindando una evaluación poco precisa (Schonhaut et al., 2003). En línea con este planteo, se ha observado un bajo nivel de concordancia entre los resultados arrojados por este subtest y pruebas fonológicas en niños con trastornos del lenguaje (Schonhaut et al., 2007). Similarmente, estudios informan un escaso valor predictivo del lenguaje sobre las competencias infantiles de lectoescritura (Heresi-Venegas \& AvariaBenaprés, 2014). Sumado a lo anterior, puede agregarse lo que aportan Angulo-Ramos y MerinoSoto (2014), quienes resaltan que el TEPSI al contar con muchos ítems o materiales a veces dificulta o entorpece la aplicación del test, pudiendo llegar a modificar los resultados o sufrir distorsiones. Esta crítica es aplicable al subtest de Lenguaje, en tanto es el más extenso del instrumento y puede ocasionar fatiga o desmotivación en los niños pequeños.

Otro dato de interés arrojado por este estudio se refiere a las diferencias encontradas en el desempeño en el TEPSI entre los niños argentinos y los chilenos, así como la variabilidad en los ritmos de crecimiento registrados por el test. Los infantes argentinos presentaron un nivel de desarrollo psicomotor superior a sus pares chilenos, siendo el área de motricidad en la cual se registraron las mayores diferencias. Una explicación posible a estos resultados radica en que 
la elaboración del TEPSI fue efectuada en 1980, registrándose la primera edición impresa de la prueba en 1985, en Chile. Posteriormente, el test ha sido objeto de revisiones y adaptaciones, inclusive hasta el año 1997 (Haeussler \& Marchánt, 1997). En otras palabras, las normas obtenidas en este trabajo se han realizado 30 años después de la creación de la prueba original y casi 18 años posteriores a la última edición del test, dejando en evidencia que los niños alcanzan competencias psicomotrices y lingüísticas con mayor celeridad que 30 años atrás.

Los enormes avances y cambios en las disciplinas científicas y tecnológicas, la globalización actual, no solo se plasmaron a nivel teórico, sino que también alcanzaron las prácticas de la vida cotidiana de las comunidades y familias (Barbero-García, Vila-Abad, \& Holgado-Tello, 2008; Díaz-Granados, 2007). El neurodesarrollo es modelado por los cambios en las prácticas cotidianas de una cultura, en tanto resulta de una delicada y continua interacción entre la maduración y la estimulación ambiental. En consecuencia, los instrumentos empleados para valorar sus hitos requieren ser actualizados en línea con dichos avances (Brenlla, 2004; Butman et al., 2001). Las diferencias halladas en el desempeño y en el ritmo del desarrollo psicomotor entre la muestra argentina y la chilena, se encuentran en línea con este planteo y señalan dos aspectos de interés. Por un lado, refieren la necesidad de actualizar los baremos chilenos del TEPSI y por otro, denuncian la importancia de disponer normas locales del instrumento.

Existe consenso respecto a que la calidad de un instrumento que evalúa la inteligencia o el nivel de desarrollo de un niño, se encuentra afectada por la calidad de las normas de interpretación de las que se disponen (Alarcón-Paz, Díaz-Valenzuela, HernándezRosales, \& Estrada-Goic, 2012). Mientras más pertinentes estas sean, en términos culturales, socioeconómicos y sociocontextuales, mayor será su capacidad para interpretar adecuadamente el resultado del niño en la prueba (Méndez-Sánchez \& Palacios-
Salas, 2001). Incluso, algunos autores señalan que el uso de normas nacionales para un determinado grupo de edad, pueden resultar inadecuadas para grupos específicos de dicha población, como por ejemplo, la población infantil vulnerable desde una perspectiva socioeconómica (Mansilla, Vásquez, \& Estrada, 2012).

En función de lo expuesto, y dada la importancia de contar con instrumentos baremados en población local, el aporte principal del estudio reside en la obtención de normas preliminares del TEPSI para preescolares mendocinos. Dichas normas resultaban inexistentes hasta la actualidad, por lo cual disponer de las mismas permitirá una adecuada interpretación de los resultados obtenidos a partir de la evaluación con el test a niños y niñas con características similares a la muestra estudiada. Se reconoce que las normas elaboradas son preliminares, vale decir, tienen un carácter precedente o inicial, por lo cual se sugiere que próximos estudios seleccionen una muestra probabilística y representativa con el fin de generar baremos para población argentina. No obstante, acordamos con Peredo-Videa (2017), sobre la importancia de evaluar sistemáticamente los instrumentos en relación a las poblaciones locales antes de promover su utilización generalizada. Estudios posteriores deberían analizar el impacto de variables ambientales en los niveles de desarrollo psicomotor, tales como nivel socioeconómico familiar, especialmente nivel de educación de los progenitores, diferencias entre niños escolarizados y no escolarizados y características de los jardines maternales, a fin de elaborar normas contextualizadas y representativas de la población meta.

Por último, se informa como dificultad de este estudio algunas características inherentes a la prueba aplicada: excesivo tiempo de prueba, numerosos ítems y materiales que componen el test, altos niveles de fatiga de niños y evaluadores. Estos aspectos deberán ser contemplados a la hora de realizar estudios normativos, y tal como sugieren algunos expertos (Angulo-Ramos \& Merino-Soto, 2014), las 
evaluaciones deberían estar a cargo de psicólogos especializados o profesionales de la salud debidamente entrenados no solo en la administración del TEPSI, sino en los principios que rigen la evaluación neuropsicológica infantil.

En suma, los datos preliminares de este trabajo quedan a la espera de su corroboración en base a nuevos estudios con muestras locales y nacionales de carácter representativos. No obstante, ante la ausencia de baremos argentinos de este instrumento, los resultados presentados en este trabajo constituyen un precedente y podrán ser utilizados para el despistaje de dificultades psicomotrices en niños mendocinos.

\section{Conclusiones}

La importancia de elaborar normas locales del TEPSI radica en que los diferentes contextos socioculturales en los cuales los niños crecen, modulan su desarrollo neurocognitivo y en consecuencia, resulta indispensable contar con referencias locales para realizar evaluaciones precisas y confiables. En Argentina, el TEPSI ha sido empleado en la evaluación y seguimiento de niños prematuros, sin embargo, no hay estudios normativos del test ni de sus propiedades psicométricas.

El presente trabajo presenta evidencia inicial sobre las propiedades de medida del test en niños argentinos, siendo su aporte principal brindar normas locales preliminares para infantes de 3 a 5 años de edad. Esto permitirá que profesionales de la salud puedan contar con una base de referencia y comparación actualizada para evaluar el desarrollo psicomotor en infantes de Mendoza.

El TEPSI es un instrumento de despistaje reconocido y empleado en diversos países latinoamericanos. Los datos arrojados por este instrumento se utilizan para diagramar diagnósticos en profundidad, para predecir dificultades ulteriores en el desarrollo cognitivo y motor de los niños, dificultades de aprendizaje y como herramienta para el seguimiento de intervenciones. En consecuencia, se espera que estudios futuros puedan contrastar y ampliar los datos arrojados por esta investigación, en miras a construir baremos nacionales de este instrumento.

\section{Conflicto de intereses}

Los autores aclaran que no existe ningún tipo de conflicto de interés para que el manuscrito sea sometido a evaluación.

\section{Responsabilidad ética}

Para la evaluación de cada niño participantes se solicitó a los padres de los mismos que firmaran un consentimiento informado que autorizara el trabajo con los menores. En dicha autorización se describía el procedimiento que se realizaría para evaluar a los niños como así también los materiales a utilizar. No obstante, también se tuvo en cuenta el asentimiento del niño para trabajar. Al finalizar las evaluaciones se entregó a los padres e instituciones un informe de devolución de cada niño con los aspectos evaluados y sus resultados.

El Comité de ética del Instituto de Ciencias Humanas, Sociales y Ambientales (INCIHUSA), Centro Científico Tecnológico, Consejo Nacional de Investigaciones Científicas y Técnicas- MENDOZACONICET (Argentina), manifestó su aprobación al proyecto de trabajo. En relación a la privacidad y consentimiento informado, en el presente trabajo no aparecen datos personales de los participantes.

\section{Agradecimiento}

El presente trabajo fue subsidiado por la Facultad de Psicología de la Universidad del Aconcagua, Mendoza (Argentina), y por la Comisión Nacional Salud Investiga del Ministerio de Salud de la Nación Argentina, a través de su programa de Becas de Perfeccionamiento en Salud Pública Carrillo-Oñativia. 


\section{Referencias}

Aguinaga-Espinoza, H. (2012). Desarrollo psicomotor en un grupo de estudiantes de 4 años de educación inicial de la red 06 Callao (Tesis de Maestría). Recuperada de http://repositorio.usil.edu.pe/handle/ $123456789 / 1079$

Alarcón-Paz, C., Díaz-Valenzuela, V., Hernández-Rosales, J., \& Estrada-Goic, C. (2012). Estudio sobre la pertinencia del uso de las normas disponibles del Raven en adultos mayores chilenos. Psico-USF, 17(3), 387-395.

Anastasi, A., \& Urbina, S. (1998). Tests Psicológicos. México: Prentice Hall.

Angulo-Ramos, M., \& Merino-Soto, C. (2012). Propiedades de medición del inventario de despistaje preescolar de Minneapolis (IDPM). Revista de enfermería Herediana, 5(2), 114-124.

Angulo-Ramos, M., \& Merino-Soto, C. (2014). TEPSI en cuestión: ¿usarlo mejor o reemplazarlo? Revista de enfermería Herediana, 7(2), 107-110.

Astete, J., Gastañaga, M., Fiesta, V., Oblitas, T., Sabastizagal, I., Lucero, M., ... Suarez, M. (2010). Enfermedades transmisibles, salud mental y exposición a contaminantes ambientales en población aledaña al proyecto minero Las Bambas antes de la fase de explotación, Perú 2006. Rev Perú Med Exp Salud Pública, 27(4), 512-519.

Atalah, E., Cordero, M., Guerra, M. E., Quezada, S., Carrasco, X., \& Romo, M. (2014). Monitoreo de los indicadores del Programa «Chile Crece Contigo» 2008-2011. Revista Chilena de Pediatría, 85(5), 569-577.

Barbero-García, M., Vila-Abad, E., \& Holgado-Tello, F. (2008). La adaptación de los tests en estudios comparativos. Acción psicológica, 5(2), 7-16.

Brenlla, M. (2004). Aspectos socio culturales y métricos en la adaptación de tests: un studio en base al test de inteligencia para dultos de Wechsler III (WAIS III). XI Jornadas de Investigación de la Facultad de Psicología de la Universidad de Buenos Aires, Buenos Aires. Recuperado de http://docplayer.es/23453801Xi-jornadas-de-investigacion-facultad-de-psicologiauniversidad-de-buenos-aires-buenos-aires-2004.html
Butman, J., Arizaga, R. L., Harris, P., Drake, M., Baumann, D., de Pascale, A., \& Ollari, J.A. (2001). El «MiniMental State Examination» en español. Normas para Buenos Aires. Revista de Neurología Argentina, 26(1), 11-15.

Calero, M., Carles, R., Mata, S., \& Navarro, E. (2010). Diferencias en habilidades y conducta entre grupos de preescolares de alto y bajo rendimiento escolar. Relieve, 16(2), 1-17. Recuperado de http://www.uv.es/ RELIEVE/v16n2/RELIEVEv16n2_5.htm

Canetti, A., Cerutti, A., Navarrete, C., Schwartzmann, L., Roba, O., \& Zubillaga, B. (2002). Sobre desarrollo infantil de niños/as menores de 5 años $y$ características familiares, en condiciones de pobreza. Conferencia presentada en el Seminario Relaciones entre el Contexto, Familia e Infancia en la Sociedad Contemporánea, Montevideo, Uruguay. Recuperado de http://www.iin.oea.org/conferencia_ ana_ceruti.htm

Castro-Solano, A., \& Fernández-Liporace, M. (2017). La evaluación psicológica en niños. Técnicas de screening y diagnóstico. Buenos Aires: Paidós.

Coe, R., \& Merino-Soto, C. (2003). Magnitud del Efecto: Una guía para investigadores y usuarios. Revista de Psicología de la PUCP, 21(1), 145-177.

Cohen, J. (1992). Statistical power analysis. Current Directions in Psychological Science, 1, 98-101. doi: 10.1111/1467-8721.ep10768783

Cova, F., Valdivia, P., \& Maganto, M. (2005). Diferencias de género en psicopatología en la niñez: Hipótesis explicativas. Revista Chilena de Pediatría, 76(4), 418-424. doi: 10.4067/S0370-41062005000400014

Díaz-Granados, F. (2007). Los niños y las familias frente a las Tecnologías de la Información y las Comunicaciones. Psicología desde el Caribe, 20, 208-224. Recuperado de http://ciruelo.uninorte.edu.co/ pdf/psicologia_caribe/20/9_Los\%20ninos\% 20y\%20las\%20familias.pdf

Flores, S. (2013). La importancia de las pruebas para evaluar el neurodesarrollo de los niños. Boletín Médico del Hospital Infantil de México, 70(3), 175-177. 
Garaigordobil, M., \& Amigo, R. (2010). Inteligencia: diferencias de género y relaciones con factores psicomotrices, conductuales y emocionales en niños de 5 años. Interdisciplinaria, 27(2), 229-245.

George, D., \& Mallery, P. (2011). IBM SPSS Statistics 21 step by step: A simple guide and refer-ence $\left(13^{\text {th }}\right.$ ed.). Boston: Pearson Education.

Gerometta, G. V., Aspres, N., Schapira, I., Granovsky, G., \& Vivas, S. (2014). Evaluación a largo plazo del desarrollo psicomotor e intelectual de niños de 4 a 5 años de edad nacidos pretérmino y con muy bajo peso. Revista del Hospital Materno Infantil Ramón Sardá, 33(1), 2-7.

Haeussler, I., \& Marchánt, T. (1997) TEPSI: Test de Desarrollo Psicomotor 2-5 años. Chile: Editorial Universidad Católica de Chile.

Heresi-Venegas, C., \& Avaria-Benaprés, A. (2014). El resultado obtenido en el TEPSI a los 4 años como herramienta predictiva del aprendizaje de la lectura en primero básico. Revista Pediatría Electrónica, 11(3), 2-9. Recuperado de http://www.revistapediatria .cl/volumenes/2014/vol11num3/pdf/TEPSI.pdf

Hernández, R., Fernández, C., \& Baptista, P. (2006). Metodología de la Investigación. México: McGrawHill.

Jiménez-Capa, P. (2014). Prevalencia de alteraciones del desarrollo psicomotor en niños de edad preescolar. Propuesta de una guía para la aplicación del test de TEPSI año 2010 (Tesis de Maestría). Recuperada de http://repositorio.ug.edu.ec/ handle/redug/9788

Lejarraga, H. (2008). PRUNAPE: pesquisa de trastornos del desarrollo psicomotor en el primer nivel de atención. Archivos Argentinos de Pediatría, 106(2), 119-125.

Lipina, S. (2006). Vulnerabilidad social y desarrollo cognitivo. Buenos Aires: Baudino Ediciones.

Mansilla, C., Vásquez, D., \& Estrada, C. (2012). Pertinencia normativa del Raven para la evaluación de población infantojuvenil socialmente vulnerable. Terapia Psicológica, 30(1), 73-80.

Mazzoni, C., Stelzer, F., Cervigni, M., \& Martino, P. (2014). Impacto de la pobreza en el desarrollo cognitivo. Un análisis teórico de dos factores mediadores. Liberabit, 1(20), 93-100. Recuperado de http://revistaliberabit. com/es/revistas/RLE_20_1_impacto-de-la-pobrezaen-el-desarrollo-cognitivo-un-analisis-teorico-de-dosfactores-mediadores.pdf

Meisels, S., \& Provence, S. (1989). Screening and Assessment: Guidelines for Identifying Young Disabled and Developmentally Vulnerable Children and Their Families. Washington: ERIC.

Méndez-Sánchez, C., \& Palacios-Salas, P. (2001). Estandarización del test de Matrices Progresivas de Raven, escala avanzada (MPA). México: Paidós.

Merino-Soto, C. (2011). Exploración de diferencias normativas en el sistema de calificación cualitativa para el test gestáltico de bender modificado. Liberabit, 17(2), 199-209. Recuperado de http://revistaliberabit. com/es/revistas/RLE_17_2_exploracion-de-diferen cias-normativas-en-el-sistema-de-calificacion-cualitati va-para-el-test-gestaltico-de-bender-modificado.pdf

Merino-Soto, C. (2014a). Evidencias psicométricas para una batería de habilidades de aprendizaje para el despistaje del rendimiento académico en primer grado de primaria (Tesis de Maestría). Recuperada de http://www.repositorioacademico. usmp.edu.pe/handle/usmp/1338

Merino-Soto, C. (2014b). El tamaño sí importa: sobre las diferencias interculturales en la visomotricidad. Liberabit, 20(2), 373-374. Recuperado de http://revis taliberabit.com/es/revistas/RLE_20_2_el-tamano-siimporta-sobre-las-diferencias-interculturales-en-lavisomotricidad.pdf

Ministerio de Salud (2017). Norma Técnica de Salud para el Control de Crecimiento y Desarrollo de la Niña y el Niño Menor de Cinco Años. Recuperado de h t t p s : / / w w w. uni cef.org / peru/s panis h / NORMA_CRED.pdf

Myers, R., \& Martínez, J. F. (2003). Educación Preescolar en Dos Pueblos de la Mixteca Alta: Un Estudio Comparativo. Un informe presentado a UNICEF y a la Secretaría de Educación Pública. Dirección General de Investigación Educativa. México: Hacia una Cultura Democrática, A. C. 
Oiberman, A., Paolini, C., \& Mansilla, M. (2012). Escala Argentina de Inteligencia Sensorio-Motriz (EAIS): Percentiles nacionales. Interdisciplinaria, 29(2), 305323.

Orozco-Hormaza, M., Sánchez-Ríos, H., \& CerchiaroCeballos, E. (2012). Relación entre desarrollo cognitivo y contextos de interacción familiar de niños que viven en sectores urbanos pobres. Universitas Psychologica, 11(2), 427-440.

Otárola-Suárez, M. (2012). Desarrollo psicomotor según género en niños de 4 años de una institución educativa del Callao-Cercado (Tesis de Maestría). Recuperada de http://repositorio.usil.edu.pe/handle/ 123456789/1216

Papalia, D., Wendkos Olds, S., \& Feldman, R. (2009). Psicología del Desarrollo (11 ${ }^{\mathrm{a}}$ ed.). México: Mc Graw Hill.

Peredo-Videa, R. (2017). Propiedades psicométricas del test de madurez mental de California. Revista de Investigacion Psicológica, 17, 11-28.

Portellano, J. A. (2007). Neuropsicología Infantil. Madrid: Síntesis.

Razali, N. M., \& Wah, Y. B. (2011). Power comparisons of Shapiro-Wilk, Kolmogorov-Smirnov, Lilliefors and Anderson-Darling tests. Journal of Statistical Modeling and Analytics, 2(3), 21-33.

Romero, E. (2011). Confiabilidad y validez de los instrumentos de evaluación neuropsicológica. Subjetividad y Procesos Cognitivos, 15(2), 83-92.
Schapira, I. (2007). Comentarios y aportes sobre desarrollo e inteligencia sensorio-motriz en lactantes. Análisis de herramientas de evaluación de uso frecuente. Revista del Hospital Materno Infantil Ramón Sardá, 26(1), 2127.

Schapira, I., Aspres, N., Benítez, A., Vivas, S., Rodríguez, G., Gerometta, G., \& Fiorentino, A. (2008). Desarrollo alejado de niños nacidos prematuros. Revista del Hospital Materno infantil Ramón Sardá, 27(4), 155158.

Schonhaut, L., Rojas, P., \& Kaempffer, A. (2003). Factores de riesgo asociados a déficit del desarrollo psicomotor en preescolares de nivel socioeconómico bajo. Comuna urbano rural, Región Metropolitana. Revista Chilena de Pediatría, 6(76), 589-598. doi: 10.4067/ S0370-41062005000600006

Schonhaut, L., Maggiolo, L., Barbieri, O., Rojas, P., \& Salgado, A. (2007). Dificultades en el lenguaje en preescolares: Concordancia entre el test TEPSI y la evaluación fonoaudiológica. Revista Chilena de Pediatría, 78(4), 369-375. doi: 10.4067/S0370-4106 2007000400004

Tornimbeni, S., Pérez, E., \& Olaz, F. (2008). Introducción a la Psicometría. Buenos Aires: Paidós.

Vericat, A., \& Orden, A. (2010). Herramientas de Screening del Desarrollo Psicomotor en Latinoamérica. Revista chilena de pediatría, 81(5), 391-401. doi: 10.4067/ S0370-41062010000500002 
Adriana V. L. Espósito

Universidad del Aconcagua (Argentina)

Doctora en Psicología, Docente e Investigadora. Coordinadora de gestión del Instituto de Investigaciones de la Facultad de PsicologíaUDA (Mendoza, Argentina). Labor clínica en el área de neuropsicología. Su línea de investigación se ha enfocado en en el área de neuropsicología infantil en relación a la aplicación de programas de intervención en estimulación cognitiva y al estudio y adaptación de pruebas neuropsicológicas para la edad infantil.

ORCID: 0000-0001-8332-0509

Autora corresponsal: avlesposito@gmail.com

Celina Graciela Korzeniowski

Universidad del Aconcagua (Argentina)

Doctora. en Psicología, cuenta con investigaciones en funciones ejecutivas, programas de estimulación cognitiva, relaciones entre funciones cognitivas, rendimiento escolar y pobreza. Su línea de investigación está centrada en evaluación y estimulación neurocognitiva infantil.

ORCID: 0000-0001-6678-3472

celinakorze@gmail.com

Marina Santini Bertoldi

Universidad del Aconcagua (Argentina)

Licenciada en Psicología, diplomada en Psicología clínica conductual cognitiva integrativa. Participación en varios proyectos de investigación del Instituto de Investigaciones de la Facultad de Psicología de la Universidad del Aconcagua, orientados en la temática de desarrollo neurocognitivo en la infancia; actualmente desempeñando funciones en la Dirección de Niñez, Adolescencia y Familia del Gobierno de Mendoza. Expositora en Congreso Latinoamericano para el avance de la Ciencia Psicológica, año 2016, sobre normas preliminares del Test de Desarrollo Psicomotor TEPSI.

marinasantini.b@gmail.com 


\section{Anexo}

Porcentaje de niños con un desempeño normal, en riesgo y retraso en el TEPSI

\begin{tabular}{ccccc}
\hline Rendimiento & Coordinación & Lenguaje & Motricidad & Total TEPSI \\
\hline Normal & $77.5 \%$ & $83 \%$ & $80 \%$ & $83.5 \%$ \\
Riesgo & $18.9 \%$ & $14.9 \%$ & $15.4 \%$ & $14.4 \%$ \\
Retraso & $3.5 \%$ & $1.99 \%$ & $4.47 \%$ & $1.99 \%$ \\
\hline
\end{tabular}

